\title{
A New Generation of Throat Cleaning and Healing Filmogen Solution for the Treatment of Productive (Wet) Cough
}

Nathalie Cucuat ${ }^{1 *}$, Sara Adel Adly ${ }^{2}$, Dankwart Rauscher ${ }^{3}$, Joydeep Mukerji ${ }^{4}$, Laurent Hamelin ${ }^{1}$ and Ravi Shrivastava ${ }^{1}$

${ }^{1}$ Vitro-Bio Research Institute, ZAC de Lavaur, Issoire, France

${ }^{2}$ AMC pharmaceuticals, Alexandria, Egypt

${ }^{3}$ Head of Portfolio and Licensing Management, Recordati Pharma, Ulm, Germany

${ }^{4}$ Wockhardt Bio AG, Dubai, UAE

\begin{abstract}
Objective: Wet cough is one of the most common pathologies in the world affecting nearly $12 \%$ of the world population. Being a multifactorial disease, involving initially viral infection, followed by bacterial secondary superinfection, damage to the throat mucosa, production of sticky mucus, and poor local immunity, there is currently no effective drug available. We conceived VB-Gy, an osmotically active filmogen solution, capable of cleaning the infected surface of all contaminants topically present, and of preparing a favorable ground for healing. The efficacy and safety of VB-Gy is assessed through a clinical trial, vs. saline solution as comparator product.
\end{abstract}

Methods: The study was a multi-centric, randomized, placebo-controlled, double-blind trial on patients suffering from wet cough. The study involved 39 in the test product group and 15 patients in the comparator group, each patient applying 3-4 sprays of investigational product 4-5 times per day, over a maximum period of 15 days. The effects on wet cough severity, frequency, chest discomfort, as well as on throat pain, irritation, requirement for antibiotics, and answers to Leicester cough questionnaire for quality of life, were evaluated.

Results: Although regular and frequent use of saline solution spray helped reduce wet cough symptoms noticeably, the test product induced very fast and nearly total regression of all clinical signs without any side effects, with statistically highly significant difference compared to placebo comparator and baseline data.

Conclusion: Treating wet cough requires a multi-target, cell-friendly, and safe approach to obtain not only symptomatic but also curative relief. VB-Gy is a completely new generation of nearly instant, safe, and multi-target therapeutic approach of treating various topical infections.

Keywords: Wet cough; Infection; Mucus; Instant; Clinical; Curative treatment

\section{Introduction}

Coughing is a reflex response to irritation of the airways in the lungs. Cough is primarily linked with the upper respiratory tract infection, though there are other conditions which can build cough. Upper respiratory tract (URT) viral and bacterial infections, common cold, asthma, and smoke are the most common causes of cough. Cough is considered chronic when it remains more than three weeks [1].

Cough can be primarily differentiated into two main types; i.e. the dry or tickly cough (without phlegm) and the wet, chesty or productive cough (with phlegm/mucus). Dry cough can again be categorized into three types; one is when there is just an itchy feeling in the throat caused by the viral infection of throat and nose, second is when there is infection in the larynx and there is a barking cough or croup, while the third type of dry cough is called whooping cough where the affected person continuously coughs with a whooping sound [2]. Such type of dry cough is caused by microbial infection while wet cough is usually caused by an initial viral infection followed by destruction of URT mucosa and subsequent inflammation. Microbial growth may also cause pus formation in the URT, leading to strong secretion of mucus and wet coughing. When URT mucosa is damaged, mucus becomes thick and sticky [3].

Dry cough is generally treated with antitussives while wet cough is treated with an expectorant. The antitussives help symptomatically suppress the cough reflex, while the expectorants are used to soften and expel the cough [4]. The aim of using expectorants is to dilute and make the mucus thinner for easy expulsion. Unfortunately, all the expectorants such as ipecacuanha, and guaifenesin are known to cause multiple side effects, particularly nausea and vomiting, and their efficacy is highly debatable [5-7]. All other currently available treatments for wet cough are symptomatic as antiviral drugs are recommended only for serious cases while antibiotics, antipyretics, anti-inflammatory drugs, and decongestants are either inappropriate, only act symptomatically, or have multiple side-effects [8-10].

An ideal treatment for wet cough should not only liquify and remove the mucus but should also remove contaminants from the URT mucosa, should act as an antiseptic, and be non-toxic and cell friendly to let the mucosa cells regenerate, repair the damage and resume their normal defense functions [11].

As a multi-target treatment approach, we conceived a new glycerolbased solution (VB-Gy), nearly 18 times more osmotically active than sea water yet not as irritant [12]. VB-Gy was rendered filmogen (F-VBGy) by incorporating specific polymeric ingredients (CW-cyanidins) to improve film flexibility and resistance to hypotonic liquid flow. Its resistance against hypotonic liquid flow was further improved by adding specific hydrophobic essential oils [13]. It was postulated that being highly osmotic, F-VB-Gy should exert sufficient osmotic pressure over semi-permeable URT mucosa to create an outward flow

*Corresponding author: Nathalie Cucuat, VITRO-BIO Research Institute, Issoire France, Tel: +33 [0]4 735505 05; E-mail: n.cucuat@vitrobio.com

Received July 11, 2017; Accepted July 17, 2017; Published July 21, 2017

Citation: Cucuat N, Adly SA, Rauscher D, Mukerji J, Hamelin L, et al. (2017) A New Generation of Throat Cleaning and Healing Filmogen Solution for the Treatment of Productive (Wet) Cough. J Pulm Respir Med 7: 415. doi: 10.4172/2161105X.1000415

Copyright: $\odot 2017$ Cucuat N, et al. This is an open-access article distributed under the terms of the Creative Commons Attribution License, which permits unrestricted use, distribution, and reproduction in any medium, provided the original author and source are credited. 
of hypotonic liquid, strong enough to mechanically remove all the contaminants without being irritant or cytotoxic.

The clinical efficacy and safety of this new generation of filmogen, osmotically active solution, was evaluated in a comparative, randomized, double blind, observational clinical trial, versus saline spray as placebo, on patients suffering from wet cough.

\section{Materials and Methods}

\section{Location}

This clinical trial was conducted at Nexus Clinical Research Center in India, affiliated to Nexus Clinical Research LLC, USA. The protocol and the study design were approved by the Institutional Ethical Committee of India - Rajiv Gandhi Institute of medical sciences (EC Registration No. ECR/492/Inst/AP/2013, dated 05/12/2013) and the trial was conducted following the ICH-GCP guidelines as per the declaration of Helsinki concerning ethical principles for medical research involving human subjects.

\section{Test and comparator products}

The test product (TP) consisted in a viscous solution of F-VBGy filmogen glycerol containing CW-Cyanidin polymeric premix, presented in a $30 \mathrm{ml}$ aluminum container with a spray. The comparator product $(\mathrm{CP})$ was presented identically, with a different code number, and contained saline solution $(0.9 \% \mathrm{NaCl})$ as placebo.

\section{Study design and objectives}

The study was designed as a multi-centric, randomized, placebocontrolled, double blind trial to assess the efficacy and safety of test product $(\mathrm{TP})$ and comparator product $(\mathrm{CP})$ on patients suffering from wet cough.

\section{Duration of the study}

Duration of the study is 15 days.

\section{Number of patients planned}

Minimum 15 in the CP group and 36 in the TP group.

\section{Doses and the rationale for the selection of doses}

During a pilot clinical trial with F-VB-Gy throat spray, the product was applied as a spray solution over the throat surface every 30 minutes during the first 2 hours, in the beginning of the treatment, and 3-4 times per day thereafter, up to complete recovery or for a maximum period of 15 consecutive days. As this mode of application was found relatively effective and was well tolerated by the patients, the same posology was used for this trial $[14,15]$.

\section{Rationale for selecting saline solution as CP}

Saline solution has no side effects, it can be used as a spray, it is colorless and odorless, cell-friendly, non-chemical, and considered as one of the most effective and safest treatments to clean infected biological surfaces $[16,17]$. As the TP's physical properties were close to those of the saline solution (with the exception of higher osmotic properties), the investigators proposed saline as CP.

\section{Rationale for accepting concomitant antibiotherapy}

The use of antibiotics to treat wet cough of viral or bacterial origin is very common in Europe but also in developing countries like India, creating a strong concern over antibiotic resistance [18]. This is the reason why investigators were allowed to use antibiotics, if required in serious cases, so as to evaluate the products' impact on the necessity for antibiotics.

\section{Rationale for selecting the number of patients}

To obtain sufficient data having statistical significance, the statistician of Nexus clinical research advised to obtain at least 15 exploitable results in the $\mathrm{CP}$ group and 30 in the $\mathrm{TP}$ group.

\section{Rationale behind selecting the study population}

In the absence of safety data on TP's use in children and pregnant women, children below 8 and pregnant women were excluded from this study.

\section{Inclusion and exclusion criteria}

Main inclusion criteria were: Male and female patients, aged between 08 and 65 years; diagnosed as having recent severe wet (productive) cough of any origin, with related symptoms such as throat pain, difficulty swallowing, throat redness, throat irritation/itching; not under any antibacterial or antiviral treatment before recruitment; ready to follow the protocol and to abstain from using any product during the study period which would have affected the study outcome.

The key exclusion criteria were: Hypersensitivity or allergy to any of the investigational products components; patients who took any medication which may affect study outcome within the last 72 hours before screening; participants with unproductive cough (dry cough); patients with diagnosis of diseases of the lower respiratory tract LRT: inflammation of the larynx, trachea, bronchitis, pneumonia, asthma, sinusitis, allergic rhinitis, as well as heart disease; under antibiotic treatment during the previous 14 days; young population below 8 years of age, and pregnant women.

\section{Randomization process}

After screening, patients satisfying all the inclusion criteria were enrolled and randomly allocated in 2:1 ratio as per randomization schedule to receive TP or CP. Treatments were allocated to patients by carrying out randomization using SAS Version 9.1.3 following a randomization schedule. Block Randomization methodology was employed for generating the list. Within the block the treatments were distributed in the ratio of $2: 1$.

\section{Product Administration}

For TP and CP products alike, patients were asked to spray (4-5 actuations at a time) the solution over the throat surface every 30 minutes during the first 2 hours and then for 3-4 times on day 1 , and 4-5 times per day from day 2 to day 15 or until recovery (whichever was earlier).

\section{Parameters studied}

The primary parameters studied were the effects on wet cough severity, frequency, chest discomfort score, and change in the color and consistency of sputum. Parameters were recorded at screening, 2 hours after the start of the treatment and thereafter on day 1, day 2, day 3 , day 6 , day 9 , day 12 , day 15 or up to complete recovery (whichever was earlier), and the changes were compared to baseline (T0: start of treatment) and to CP scores.

Key secondary parameters included effect on throat pain, throat irritation, and throat redness. All parameters were evaluated individually on a $1-10$ scoring scale $(0=$ normal and $10=$ worst condition $)$ by the investigator at the start and at the end of the study or by the patient during the study. The need for antibiotic treatment during the 
study, product acceptability by the patients and the investigators, as well as the impact on the quality of life of the patients as per Leicester Cough Questionnaire-Acute [19], were also evaluated at the screening visit and on day 15 . Safety assessment: at the end of the study, subjects and investigators evaluated any eventual treatment-emergent adverse effects.

\section{Statistical analysis of results}

The analyses were conducted with Microsoft Excel and XLStat using the available data. Significant effects were those with a probability lower than $\alpha=0.05$. Statistically significant results were indicated in abbreviated manner: SS and not statistically significant results ( $>0.05$ ): NS. For each score, repeated measures analysis of variance (RMANOVA) determined differences in symptoms scores across study visits. Nemenyi post-hoc test provided pairwise comparisons in a group between baseline and the end of the study. Results were compared with baseline values (scores obtained just before $1^{\text {st }}$ treatment on day 1 ) in the same group and with the $\mathrm{CP}$, at each time point.

\section{Results}

\section{Demographics}

63 subjects were screened, 5 subjects had screen failures and 58 subjects were allocated to TP group $(n=39)$ or CP group $(n=19)$. Among these patients, 39 in the TP and 15 in the CP group completed the study as shown in the flow chart (Figure 1).

At the time of recruitment, all enrolled subjects were diagnosed with acute productive cough including major symptoms such as throat pain, difficulty swallowing, throat redness, throat irritation/itching and fever (if present).

Patients with diagnosis of diseases of the lower respiratory tract: inflammation of the larynx, trachea, bronchitis, pneumonia, asthma, sinusitis, allergic rhinitis, as well as heart disease; patients who had taken medications with demulcent properties or, on the contrary, with known cough promoting side effects; patients who had used any local anaesthetic within the past 24 hours, who have used a longer acting or slow release analgesic during the previous 24 hours, having taken antibiotics during the previous 14 days or with a history of severe renal impairment or hepatic impairment were not enrolled in the study. The baseline mean symptom scores were fairly identical in both groups, with mean severity often slightly higher in the TP group at study outset.

\section{Effect on wet cough severity}

The mean values of wet cough severity were not affected in the CP group up to Day 6. Slight reduction ( $-5.0 \%, \mathrm{NS}$ : $\mathrm{p}=1.000)$ was observed on Day 9 , and the mean severity score of wet cough then decreased rapidly up to Day $12(-25.0 \%$, NS: p $>0.6)$ and Day 15 (-50.0\%, SS: $\mathrm{p}<0.0001$ ) compared to the starting values (Figure 2).

These results show that the $\mathrm{CP}$ product has no significant effect on wet cough severity during the $1^{\text {st }}$ week of treatment and little effect during the $2^{\text {nd }}$ week, as the reduction observed is considered to be related to the natural healing process and the use of antibiotics in nearly $86 \%$ patients in this group (compared to only $16 \%$ in the TP group) and not to the healing properties of the $\mathrm{CP}$.

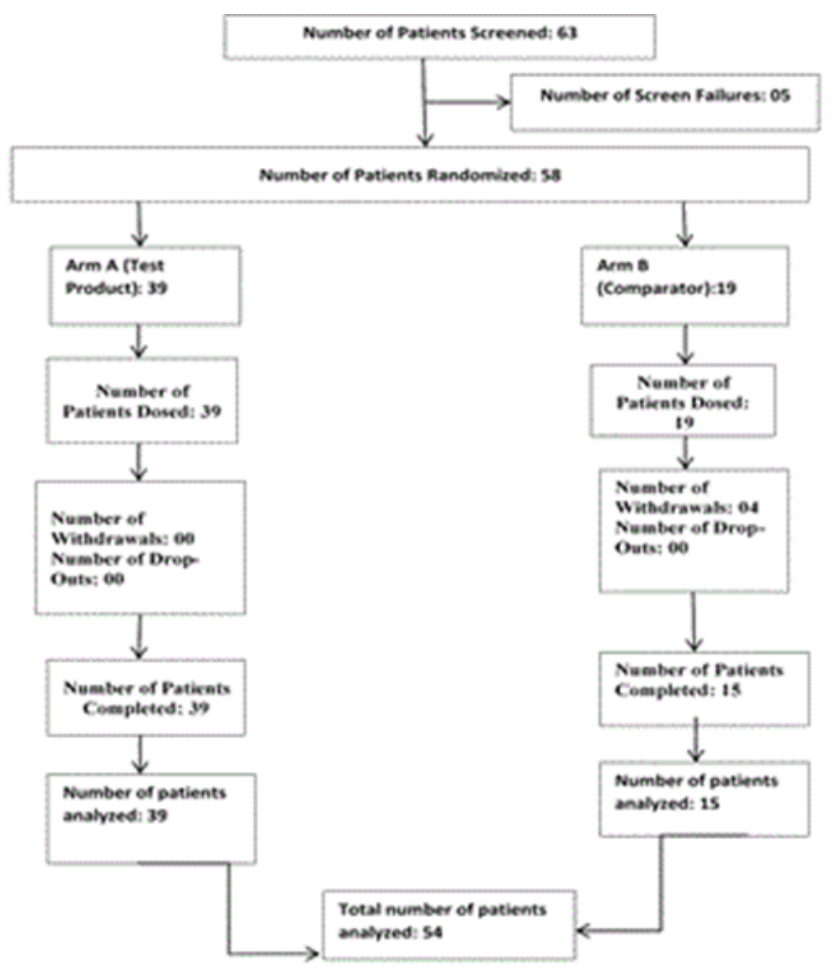

Figure 1: Flow chart of the population screened, randomized, withdrawn, and analyzed at the end of the study.

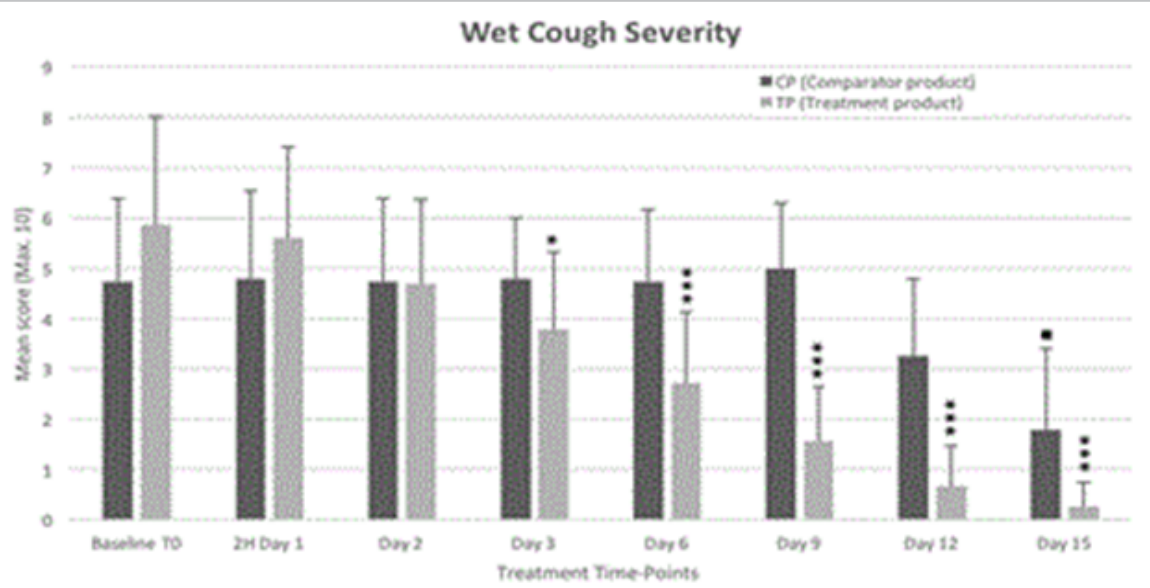

Figure 2: Mean scores ( \pm SD) for wet cough severity in TP group (gray) versus CP group (black) just before treatment (baseline T0) and on days $1(2 \mathrm{~h}), 2,3,6,9,12$ and 15. ${ }^{*} p<0.05,{ }^{* *} p<0.01, "{ }^{* *} p<0.001$ for TP compared to CP at the same time point, and $\cdot<0.05$ compared to baseline values. 
In the TP group, the mean scores for wet cough severity decreased noticeably just 2 hours after the $1^{\text {st }}$ treatment $(-5.0 \%)$ indicating that the TP helps ease wet cough, certainly by fluidizing mucus due to its mode of action, and thus facilitating its expulsion, as soon as it is applied over the throat surface. This reduction observed after 2 hours is slight and not statistically significant $(\mathrm{p}=1.00)$ which is comprehensible as the observations were recorded very rapidly after the $1^{\text {st }} \mathrm{TP}$ administration, where only minor reduction in the intensity of wet cough severity coupled with individual variations considerably affect the statistical significance. We consider that these effects are related to the administration of TP because the reduction was much stronger $(-20.0 \%, \mathrm{NS}: \mathrm{p}=0.2)$ on day 2 and continued steadily and with statistical significance $(\mathrm{p} \leq 0.002)$ on Days $3(-36.0 \%), 6(-54.0 \%), 9$ $(-75.0 \%), 12(-85.0 \%)$ and $15(-91.0 \%)$. This reduction in the severity in the TP group is highly significant from day 3 onward compared to the $\mathrm{CP}$ group $(\mathrm{p}<0.05)$ and compared to starting score $(\mathrm{p} \leq 0.002)$, and is therefore considered to be directly related to the effects of the TP.

\section{Effect on wet cough frequency}

The mean values of wet cough frequency show that the reduction in cough frequency was significantly more important in the TP group right from the $2^{\text {nd }}$ day of treatment (day 3, SS: $\mathrm{p}=0.009$ ) (Figure 3). In the $\mathrm{CP}$, the mean score of wet cough frequency started diminishing slightly but non-significantly ( $p>0.5$ ) from day $2:-19.0 \%$ on day 12 with more marked and significant $(\mathrm{p}<0.0001)$ reduction at the end of the study period, from days 12 to 15 . This reduction is more likely to be related to the natural healing process and the use of antibiotics than to the activity of the saline solution.

In the TP group, the frequency of cough started decreasing strongly and steadily from day 2 , with statistical significance from day 3 ( $p=0.001$ compared to baseline, and $\mathrm{p}=0.009$ compared to $\mathrm{CP}$ ) and nearly $75 \%$ reduction on day $9(\mathrm{p}<0.0001)$. These results show that wet cough lasts for at least 9-12 days before natural recovery process starts blocking the infection. The results of this study also show that although the TP helps reducing the severity of wet cough after 2 hours of $1^{\text {st }}$ treatment, it does not have as fast an effect on its frequency.

\section{Effect on chest discomfort}

In the $\mathrm{CP}$ group, the mean chest discomfort score was not reduced until day 9; on the contrary, this score increased slightly compared to day 1 (T0 baseline) values. Afterwards, probably due to natural healing and the use of antibiotics in a majority of patients, the mean score started decreasing, but not significantly ( $\mathrm{p}>0.8$ ), between days 9 and 12 , and more strongly and significantly between days 12 and $15(-58.0 \%$, $\mathrm{p}=0.001$ ) compared to the initial value (Figure 4).

In the TP group, there was a drastic and steady reduction in mean chest discomfort score compared to baseline from day 1 (with statistical significance on day $3, p=0.0002$ ) up to the end of the treatment period. It should be noted that the mean chest discomfort score was decreased by about $14 \%$ after 2 hours of the start of treatment clearly indicating that the TP starts improving chest comfort right after the $1^{\text {st }}$ administration. The effects are rapid and strong with nearly $90 \%$ improvement on day 9 (SS: $\mathrm{p}<0.0001$ ). These results show a strong efficacy of the TP in reducing chest discomfort, which may be explained by a higher capacity to clean away the mucus and other contaminants present on the throat surface.

\section{Effect on throat pain}

The throat pain was moderate but not very strong at the start of

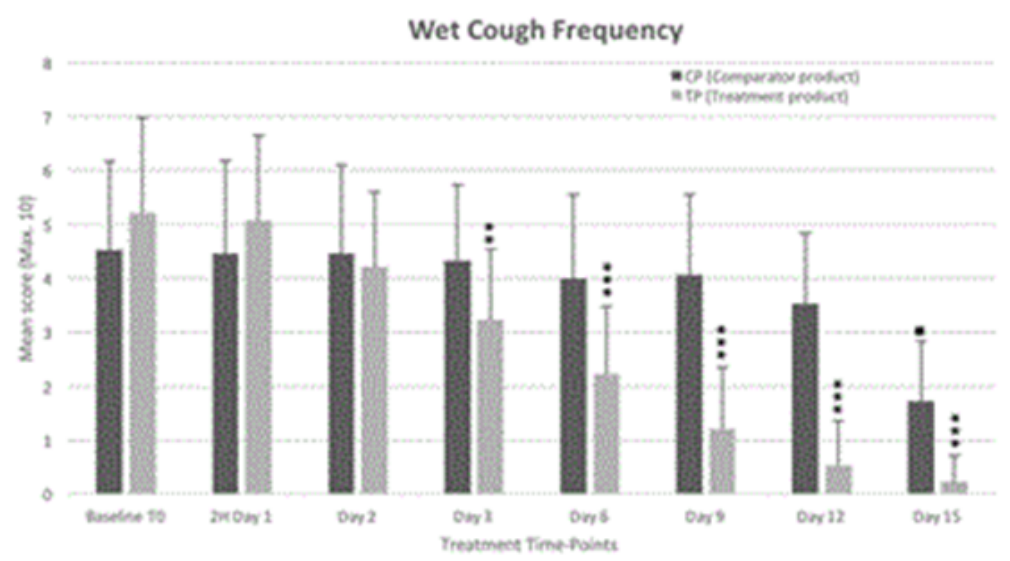

Figure 3: Mean scores ( \pm SD) for wet cough frequency in TP group (gray) versus CP group (black) just before treatment (baseline T0) and on days $1(2 \mathrm{~h}), 2,3,6,9,12$ and 15. ${ }^{*} p<0.05,{ }^{* *} p<0.01,{ }^{* * *} p<0.001$ for TP compared to CP at the same time point, and $\cdot<0.05$ compared to baseline values.

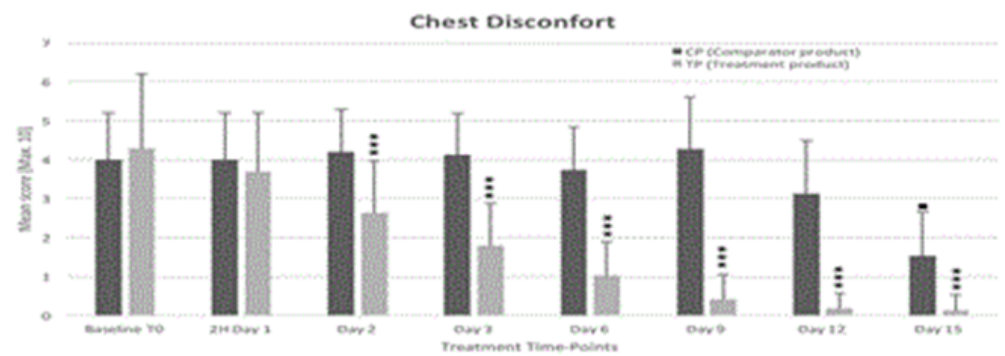

Figure 4: Mean scores ( \pm SD) for chest discomfort in TP group (gray) versus CP group (black) just before treatment (baseline T0) and on days $1(2 \mathrm{~h}), 2,3,6,9,12$ and 15. ${ }^{*} p<0.05,{ }^{* *} p<0.01,{ }^{* *} p<0.001$ for TP compared to CP at the same time point, and $\cdot<0.05$ compared to baseline values. 
treatment in both groups (Figure 5). In the CP group, throat pain was not significantly reduced until the end of the treatment period $(\mathrm{p}<0.0001$ on day 15$)$. The percentage of change from baseline was not very noticeable up to day 9 ( $-4 \%$, NS: $\mathrm{p}=0.986)$. Afterwards, a more rapid but still incomplete recovery was observed on day 12 (-24\%, NS: $\mathrm{p}=0.08)$ and day $15(-41 \%$, SS: $\mathrm{p}<0.0001)$.

The TP does not affect throat pain instantly (observations at 5 minutes of $1^{\text {st }}$ administration) but a mean reduction of $18 \%$ (NS: $\mathrm{p}=0.4$ ) was observed as early as $2 \mathrm{~h}$ after the $1^{\text {st }}$ treatment. Afterwards, throat pain started decreasing rapidly, significantly $(\mathrm{p} \leq 0.002)$ and steadily with $40 \%$ reduction on day $2 ; 60 \%$ on day $3 ; 78 \%$ on day 6 ; and between $90-94 \%$ from day 9 onwards. These results show a strong and rapid efficacy of the TP in reducing wet cough-induced throat pain.

\section{Effect on throat irritation}

Throat irritation is a symptom commonly associated with cough and URT infection. Normally, onset of all the symptoms associated with wet cough such as throat pain and irritation begins a few hours before wet cough itself appears, and the intensity and severity of all these symptoms progress during the $1^{\text {st }}$ week before the body's natural defences start acting on the cause. This is the reason why the clinical symptoms begin decreasing after 7-8 days and natural recovery occurs within 2-3 weeks (Figure 6).

The individual results for throat irritation in the CP group show that the CP has virtually no efficacy in reducing throat irritation up to days 7-9, followed by slight recovery from Day 9 onwards. Although the intensity of throat irritation was reduced in $7 / 15$ patients on Day 9, it was not changed in 4/15 patients and had even increased in $4 / 15$ patients. The limited amplitude of score change (slight and not significant, $\mathrm{p}=0.986$ ) and the diversity of results (increase or decrease) observed on day 9 lead to the conclusion that the $\mathrm{CP}$ has very little effect on throat irritation.

In the TP group, throat irritation started decreasing in almost all patients right from day 1 . The decrease was strong, significant (from day $2, p=0.0003$ ) and steady, and most of the patients had only slight throat irritation from days 6-9 onwards. almost all patients recorded scores below $2 / 10$ from day 9 . This rapid effect may have been related to the hydrating effect of the TP. The reduction was $43 \%$ stronger on day 2 (SS: $\mathrm{p}<0.05$ ), $60 \%$ on day 3 (SS: $\mathrm{p}<0.0001$ ) and $80 \%$ on day 6 (SS: $\mathrm{p}<0.0001)$, as compared to the CP values. Throat irritation was nearly absent $(-90 \%$ or more reduction, SS: $\mathrm{p}<0.0001)$ from day 12 , indicating strong anti-irritant potential of the TP.

Considering the natural healing process implied in wet cough, the results for the $\mathrm{CP}$ at the end of the treatment can be considered poor (33.3\% patients scoring zero) compared to the TP group (where $94.7 \%$ patients scored $0 / 10$ at the end of the study). These effects can be directly attributed to the $\mathrm{TP}$, as such improvement is much superior to what could be expected with just the natural healing process.

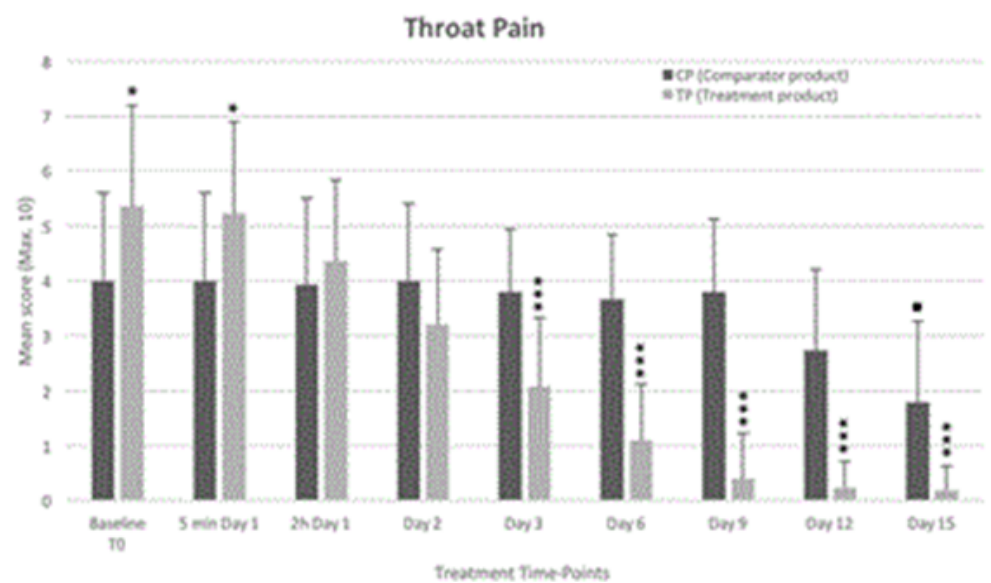

Figure 5: Mean scores ( \pm SD) for throat pain in TP group (gray) versus CP group (black) just before treatment (baseline T0) and on days $1(2 \mathrm{~h}), 2,3,6,9,12$ and 15 . $\mathrm{p}<0.05,{ }^{*} \mathrm{p}<0.01, " \mathrm{p} p<0.001$ for TP compared to CP at the same time point, and $\cdot<0.05$ compared to baseline values.

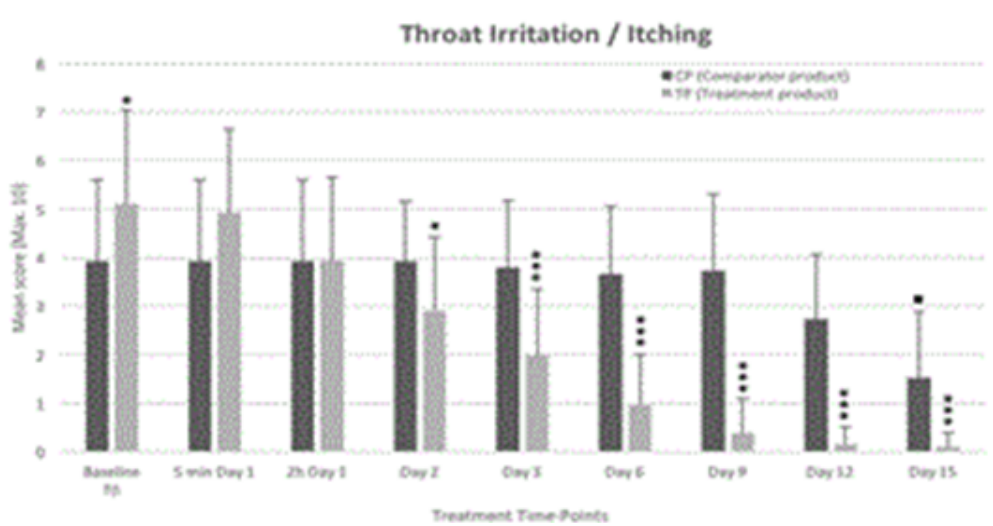

Figure 6: Mean scores ( $\pm \mathrm{SD}$ ) for throat irritation in TP group (gray) versus CP group (black) just before treatment (baseline T0) and on days $1(2 \mathrm{~h}), 2,3,6,9,12$ and 15. ${ }^{*} p<0.05,{ }^{* *} p<0.01,{ }^{* *} p<0.001$ for TP compared to CP at the same time point, and $\cdot<0.05$ compared to baseline values. 


\begin{tabular}{|c|c|c|}
\hline Parameter & CP group (n=15) & TP group (n=39) \\
\hline Number of patients & 13 & 6 \\
\hline \% of patients & $86.67 \%$ & $15.38 \%$ \\
\hline Mean duration (within the study period) & 2.54 days & 2.17 days \\
\hline
\end{tabular}

Table 1: Need for and duration of antibiotic treatment in CP and TP groups.

\begin{tabular}{|c|c|c|c|c|c|c|c|}
\hline Group & $\begin{array}{l}\text { Score at end of } \\
\text { study }\end{array}$ & $\begin{array}{l}\text { Wet Cough } \\
\text { Severity }\end{array}$ & $\begin{array}{l}\text { Wet Cough } \\
\text { Frequency }\end{array}$ & Chest Discomfort & Throat Pain & Throat Irritation & Throat Redness \\
\hline $\mathrm{CP} \%$ & $0 / 10$ & $33.33 \%$ & $20 \%$ & $26.66 \%$ & $26.66 \%$ & $33.33 \%$ & $20 \%$ \\
\hline TP $\%$ & $0 / 10$ & $76.92 \%$ & $79.49 \%$ & $89.74 \%$ & $84.62 \%$ & $89.74 \%$ & $84.62 \%$ \\
\hline $\mathrm{CP} \%$ & 0 or $1 / 10$ & $40 \%$ & $33.33 \%$ & $40 \%$ & $46.66 \%$ & $46.66 \%$ & $33.33 \%$ \\
\hline TP $\%$ & 0 or $1 / 10$ & $97.44 \%$ & $97.44 \%$ & $97.44 \%$ & $97.44 \%$ & $100 \%$ & $100 \%$ \\
\hline
\end{tabular}

NB: we also assessed the number of patients scoring either 0 or 1 at the end of the study as $1 / 10$ may also reflect a drastic, nearly complete recovery with only a hint of residual clinical sign.

Table 2: $\%$ of population in the CP $(n=15)$ and TP $(n=39)$ groups showing total recovery for individual wet cough symptoms at the end of the study.

\section{Effect on throat redness}

This symptom follows almost the same pattern of evolution as the other throat symptoms, showing that the TP affects all associated wet cough symptoms and is efficient in improving all the symptoms simultaneously. In the $\mathrm{CP}$ group, throat redness did not decrease until day 12 (NS: $\mathrm{p}=0.884$ ), and the mean value was even higher on day 9 than at baseline. Only a relatively limited decrease was observed between day 9 and end of treatment (SS: $\mathrm{p}<0.05)$. In the TP group, when mean values are compared with the starting values or with the $\mathrm{CP}$ scores, a steady decrease is seen throughout the study. $75 \%$ reduction in throat redness on day 9 (SS: $\mathrm{p}<0.0001$ ) clearly shows that this reduction is related to the administration of the TP.

\section{Effect on the sputum colour and consistency}

Approximately 36 patients had clear sputum at the end of the study. Out of these 36 patients, 35 were from the TP group and only 1 was from the CP group. Among these patients, 6 subjects had greenish sputum, 8 had yellowish sputum, 9 had thick whitish sputum, while 12 subjects who had strong yellowish sputum at visit 1 (on screening) showed normal color and consistency at the end of the study.

\section{Effect on the need for antibiotics}

Out of the 15 patients in the comparator group, 13 patients required antibiotics, whereas only 6 out of 39 patients in the TP group were prescribed antibiotics due to deteriorating condition (Table 1).

Antibiotherapy was initiated either on day 9 or on day 12 for all patients who needed antibiotics. Mean duration of treatment was comparable in both groups (around 3 days). It should be noted that the study was conducted in India, where antibiotics are generally prescribed only for 3 or 6 days.

\section{Effect on LCQ quality of life parameters}

Results indicate that the test product was very effective in improving quality of life parameters in the TP group (nearly 50\%) compared to the CP (nearly 20\%). The improvement in the LCQ score corresponds to the symptomatic improvement of all wet cough symptoms in the TP group observed in this study.

\section{Complete recovery at the end of the study}

As shown in Table 2, the symptoms of wet cough were drastically diminished in the TP compared to the $\mathrm{CP}$ group, indicating that the $\mathrm{TP}$ is capable of improving all symptoms associated with productive cough.

\section{Product acceptability}

Out of 39 patients in the TP group, 38 patients assessed their wet cough symptoms as very much improved and only 1 as minimally improved, compared to 6 patients reporting minimal and 9 reporting poor improvement in the $\mathrm{CP}$ group. Physician's assessment was comparable to the patient's.

\section{Safety and security}

Safety was assessed by evaluating the number of patients reporting incidences of adverse events (AE) and/or serious adverse events (SAE) arising during or after the study and their assessment in respect to intensity, duration, pattern and causal relationship to the investigational product. Safety was further assessed by evaluating the patient compliance diary.

The investigational products were both found to be totally safe as no treatment emergent adverse event (TEAE) was reported, nor any cases of any adverse reaction were noticed during the study. The test product was well tolerated and there were no notable changes in vital signs, physical examination, and systemic examination. F-VB-Gy is therefore considered totally safe for topical application as a throat spray for the treatment of wet cough.

\section{Discussion}

Coughing is one of the most common symptoms encountered in clinical medicine and is usually originated as an initial viral infection which progresses to the lower respiratory tract (LRT). Coughing is mediated through a reflex arc made of sensory receptors, afferent nerve fibers, a center, efferent nerve fibers, and effector muscles. The vagus is the most important afferent nerve, although the glossopharyngeal and trigeminal nerves may operate, depending on the receptors involved [18].

Viral growth damages URT and LRT mucosa, which leads to subsequent growth of opportunistic bacteria such as Streptococcus, generation of cellular debris and pus, irritation of the airways and inflammation of airway mucosa. Inflammation of the respiratory tract mucosa, from infectious or non-infectious causes, results in hyperreactivity of the cough receptors, making them more sensitive to the cough-producing effect of commonly occurring mild irritants such as cold air, respiratory pollutants, deep or fast respiration, and excessive use of the larynx [11,19]. Inflammation further stimulates mucus secretion and leads to the development of wet cough. Due to poor ciliary movements, mucus becomes thicker and sticky, and difficult to dislodge, thus generating chronic wet coughing [20-22].

Taking into consideration the physiopathology of wet cough, an ideal treatment should absolutely remove the original cause, which is viral and bacterial infection. Simultaneously, the treatment should also clean the airway passage, detach all the contaminants, liquefy the mucus 
so as to allow ciliary movements, and repair the damaged mucosa to reestablish normal airway defense functions. It is comprehensible that no drug has yet been discovered which could act on all these parameters simultaneously to treat the basic cause of wet cough $[8,23]$. Expectorants help reduce coughing by thinning the mucus in the air passages, making it easier to cough up, but without affecting the underlying cause of the coughing and acting only symptomatically, and they may have side effects such as nausea, vomiting, and occasional allergy [24]. Topical nasal decongestants reduce swelling in the nasal passages but they may cause stinging, burning and nose dryness [25]. Oral decongestants usually cause dizziness, nervousness, and difficulty sleeping. Cough suppressants are more closely related to expectorants, but instead of making mucus easier to expel, they help stop coughing altogether. Side effects associated with this medication are nausea, nervousness, drowsiness, and dizziness [26]. Antihistamines and corticosteroids are most commonly associated with allergies, but they can also be used in wet cough. The most common side effect of antihistamines is drowsiness. Natural remedies such as salt water gargling or nasal sprays help ease symptoms but require frequent and regular use over an extended period which is not always practical $[27,28]$. This shows that none of the currently available medications or remedies, chemical or natural, topical or systemic, is directed at curing wet cough, and current research is also not directed at finding a multitarget wet cough remedy [29].

F-VB-Gy is a totally a new generation of natural and mechanically acting device which surprisingly fulfills all the basic prerequisites essential to effectively treat wet cough. The results of this study clearly prove that the topical application of $\mathrm{CW}$-cyanidin-containing F-VB-Gy reduces rapidly wet cough severity, cough frequency, chest discomfort, throat pain and irritation, throat inflammation, and improves sputum consistency and quality of life of the patients. As antibiotics were generally prescribed when investigators observed aggravation associated with probable complication by secondary bacterial infection, a minimized requirement for antibiotics in the TP group indicates powerful antimicrobial properties exerted by the TP. It is postulated that cleaning the throat mucosa surface of microbial and other contaminants accelerates natural healing process and prevented bacterial surinfection. These results were not obtained with a chemical molecule, which would have produced multiple side effects nor by associating multiple approaches to ease each symptom, but simply by creating an osmotically active, non-irritant film over the damaged mucosa to help clean all the contaminants from the infected surface. When the mucosal surface is clean and free of contact with any cytotoxic chemical, natural healing begins instantly, and the mucosa resumes its normal defense functions [30]. Hypotonic liquid drawn by F-VB-Gy hypertonic film instantly fluidifies sticky mucus and detaches all the waste products present on the throat mucosa. Normalizing mucus secretion and reestablishing ciliary movements stops the cough reflex. As F-VB-Gy hyperosmotic film is prepared using food grade, dietary ingredients, and no chemical is added, applying such a product over the throat or nasal mucosa does not produce any irritation or side effects. Osmosis being an instant and mechanical process, results are nearly instant and relief starts within a few minutes after $1^{\text {st }}$ product application [31].

This technology is completely novel and safe, and can be used to treat all diseases where a live biological surface is infected, contaminated, damaged, and requires natural healing.

\section{Acknowledgements}

This clinical study was commissioned and funded by the Naturveda Laboratory.

\section{References}

1. Atkinson SK, Sadofsky LR, Morice AH (2016). How does rhinovirus cause the common cold cough? BMJ Open Respir Res 3: e000118.

2. Chung KF, Bolser D, Davenport P, Fontana G, Morice A, et al. (2009) Semantics and types of cough. Pulm Pharmacol Ther 22: 139-142.

3. Chang AB, Redding GJ, and Everard ML (2008) Chronic wet cough: Protracted bronchitis, chronic suppurative lung disease and bronchiectasis. Pediatr Pulmonol 43: 519-531.

4. Morice A, Kardos P (2016) Comprehensive evidence-based review on European antitussives. BMJ Open Respir Res 3: e000137.

5. Prabhu SS, Chandrashekharan S, Bolmall CS, Baliga V (2010) Efficacy, safety and tolerability of salbutamol + guaiphenesin + bromhexine (Ascoril) expectorant versus expectorants containing salbutamol and either guaiphenesin or bromhexine in productive cough: a randomised controlled comparative study. J Indian Med Assoc. 108: 313-314, 316-318, 320

6. Shmeleva NM (2012) Use of the mucoregulator ascoril for respiratory diseases. Ter Arkh 84: 86-90.

7. Fuloria M, Rubin BK (2000) Evaluating the efficacy of mucoactive aerosol therapy. Respir Care 45: 868-873.

8. Alloo J, Vallath S, Del Mar C, Carter M, Thorning S, et al. (2017) Determining the gaps between Cochrane reviews and trials of effectiveness of interventions for acute respiratory infections: An audit. Syst Rev. 6: 82-88.

9. Anekthananon T, Pukritayakamee S, Ratanasuwan W, Jittamala P, Werarak P et al. (2013) Oseltamivir and inhaled zanamivir as influenza prophylaxis in Thai health workers: A randomized, double-blind, placebo-controlled safety trial over 16 weeks. J Antimicrob Chemother 68: 697-707

10. Cutrera M, Baraldi E, Indinnimeo L, Del Giudice MM, Piacentini G, et al. (2017) Management of acute respiratory diseases in the pediatric population:The role of oral corticosteroids. Ital J Pediatr 43: 31.

11. Cots JM, Alós JI, Bárcena M, X Boleda X, Cañada JL, et al. (2015) Recommendations for Management of Acute Pharyngitis in Adults. Acta Otorrinolaringol Esp 66: 159-170.

12. Shrivastava $R$ (2005) Non-solid composition for local application. Patent PCT/ FR99/01340, International publication No. WO 00/74668 A1 on 14/12/2000.

13. Shrivastava R, Shrivastava L, Shrivastava R (2017) Composition for topical application comprising glycerol and tannins. International PCT application No. PCT/EP2013/061835, Publication No. WO 2014/194966 A1 of 11.12.2014.

14. Shrivastava $R$ (2011) A new therapeutic approach to neutralize throat surface proteases and virus glycoproteins simultaneously for the treatment of influenza virus infection. Int J Virol 7: 53-63.

15. Shrivastava $R$ (2011) A pilot clinical trial to evaluate the efficacy of a topical antiviral osmotically active hypertonic solution (VB-Th4) for the treatment of influenza virus induced sore throat. J Clinic Trials 1: 102.

16. Šlapak I, Skoupá J, Strnad P, Hornik P (2008) Efficacy of isotonic nasal wash (seawater) in the treatment and prevention of rhinitis in children. Arch Otolaryngol Head Neck Surg 134: 67-74.

17. Moyad MA (2009) Conventional and alternative medical advice for cold and flu prevention: What should be recommended and what should be avoided? Uro Nurs 29: 455-458.

18. Norris P, Chamberlain K, Dew K, Gabe J, Hodgetts D, et al. (2013) Public beliefs about antibiotics, infection and resistance: A qualitative study. Antibiotics (Basel) 2: 465-476.

19. Yousaf N, Lee KK, Jayaraman B, Pavord ID, Birring SS (2011) The assessment of quality of life in acute cough with the Leicester Cough Questionnaire (LCQacute). Cough 7: 4 .

20. Niimi A (2011) Cough and asthma. Curr Respir Med Rev 7: 47-54.

21. McCool FD, Leith DE (1987) Pathophysiology of cough. Clin Chest Med 8: 189-195

22. Martin MJ, Harrison TW (2015) Causes of chronic productive cough: An approach to management. Respir Med 109: 1105-1113.

23. Dicpinigaitis PV (2011) Cough: An unmet clinical need. Br J Pharmacol 163 116-124.

24. Allan GM, Arroll B (2014) Prevention and treatment of the common cold Making sense of the evidence. CMAJ 186: 190-199. 
Citation: Cucuat N, Adly SA, Rauscher D, Mukerji J, Hamelin L, et al. (2017) A New Generation of Throat Cleaning and Healing Filmogen Solution for the Treatment of Productive (Wet) Cough. J Pulm Respir Med 7: 415. doi: 10.4172/2161-105X.1000415

25. Rotter N (2016) Evidence and evidence gaps in therapies of nasal obstruction and rhinosinusitis. GMS Curr Top Otorhinolaryngol Head Neck Surg 15: Doc06.

26. Kigen G, Busakhala N, Ogaro F, Chesire E, Saat N, et al. (2015) A review of the ingredients contained in over the counter (otc) cough syrup formulations in Kenya. Are they harmful to infants? PLoS One 10: e0142092.

27. Smith SM, Schroeder K, Fahey T (2014) Over-the-counter (OTC) medications for acute cough in children and adults in community settings. Cochrane Database Syst Rev 11: CD001831.

28. Begic E, Begic Z, Dobraca A, Hasanbegovic E (2017) Productive Cough in
Children and Adolescents - View from Primary Health Care System. Med Arch 71: 66-68.

29. Piromchai $P$, Kasemsiri $P$, Laohasiriwong $S$, Thanaviratananich $S$ (2013) Chronic rhinosinusitis and emerging treatment options. Int J Gen Med 6: 453-464.

30. Klyachkina IL (2015) The new possibility for the treatment of acute cough Vestn Otorinolaringol 80: 85-90.

31. Hammel HT, Schlegel WM (2005) Osmosis and solute-solvent drag: Fluid transport and fluid exchange in animals and plants. Cell Biochem Biophys 42 : 277-345. 